\title{
Toxocara Prevalence in Soil and Humans in Vladivostok: A Long-Term
} Study

\author{
Michail Shchelkanov ${ }^{1}$, Tatyana Moskvina ${ }^{1,{ }^{*}}$, Yulia Nesterova ${ }^{2}$, Galina Zakjarova ${ }^{2}$, Khomichuk \\ Tatyana $^{2}$, Irina Galkina ${ }^{1}$ and Marina Kiseleva ${ }^{1}$ \\ ${ }^{1}$ School of Biomedicine, Far Eastern Federal University, Vladivostok, Russia \\ ${ }^{2}$ The Center of Hygiene and Epidemiology, Vladivostok, Russia \\ "Corresponding author: School of Biomedicine, Far Eastern Federal University, Vladivostok, Russia. Email: rabchan1992@gmail.com
}

Received 2018 November 23; Revised 2020 February 04; Accepted 2020 March 05.

\begin{abstract}
Background: Toxocariasis is a zoonotic parasitosis with worldwide distribution. Environmental contamination with Toxocara eggs is a main source of human infections. In Russia, particular in Vladivostok, knowledge regarding the current situation, including distribution and environmental contamination by Toxocara spp., is largely unknown.

Objectives: The present study investigated environmental contamination with Toxocara spp. eggs, in soil samples collected from sandpits and public parks in Vladivostok, Russia during 23-month period.

Methods: A total of 260 soil samples from sandpits and 155 samples from public parks were collected during July 2015 to December 2017. Soil samples were tested by sedimentation/flotation technique. Seroprevalence of Toxocara antibody in human was analyzed using Toxocara canis IgG ELISA assay.

Results: Overall, the annual prevalence of Toxocara spp. in sandpits varied between $20 \%-23 \%$ the annual prevalence of Toxocara spp. in public parks varied between $25 \%-42 \%$ in different years. The average number of Toxocara spp. varied from 0 to 220 eggs per $1 \mathrm{~kg}$ over the 3-years study period. The highest prevalence was registered in summer period. The significant correlation appeared between Toxocara spp. contamination rates in sandboxes and parks and the Toxocara prevalence in humans in 2016 year.

Conclusions: Current study demonstrates that it is necessary to promote preventive public health measures to avoid soil contamination.
\end{abstract}

Keywords: Toxocara canis, Prevalence, Public Health, Zoonosis

\section{Background}

Toxocariasis is a major zoonotic disease with worldwide distribution. It caused by nematodes from the genus Toxocara. Two species such as Toxocara canis and Toxocara cati are the main agents of human toxocariasis. Humans are the paratenic hosts for Toxocara spp., where larvae migrate and encyst in different tissues and organs. In humans, larvae cause larvae migrans syndrome. Totally four types of human toxocariasis are distinguished including ocular toxocariasis, visceral toxocariasis, covert toxocariasis (common toxocariasis) and neurotoxocariasis, due to larvae localization and clinical signs $(1,2)$.

Dogs and cats are infected Toxocara spp. worldwide, the highest prevalence often registered in puppies and kittens and free-roaming animals. The high burden of Toxocara spp. leads to vomiting, anorexia intestinal obstruction with subsequent death.

Toxocara spp. are the geohelminths, unembryonated eggs are passed with feces of definitive host in environment and then infective third stage larvae within the eggshell development within three to six weeks under appropriate conditions such as warm temperature and high humidity. So, the soil contaminated Toxocara spp. eggs is a major source of infection for humans and animals. Moreover, high contamination rates of Tocoxara spp. eggs of sandpits lead to spreading of toxocariasis among children (3-6).

\section{Objectives}

A long-term study was designed to assess the environmental contamination by Toxocara spp. eggs in soil collected from public parks and playground sandpits in Vladivostok. In addition, egg density of contaminated soil was calculated and percentage of infective third stage larvae 
was estimated. The Toxocara canis antibody prevalence in human sera was also investigated using ELISA assay.

\section{Methods}

\subsection{Study Area}

Vladivostok is a capital of Far Eastern Region. It is situated on the coast of Sea of Japan, an area characterized by a monsoonal climate, with cold and snowy winters and warm and rainy summers. The average maximum and minimum temperatures over 2009 to 2014 were $-23.9^{\circ} \mathrm{C}$ and $30^{\circ} \mathrm{C}$ respectively. Humidity levels for Vladivostok are $57 \%-60 \%$ per year. Average amount of snowfall in the winter is $47 \mathrm{~cm}$. The Vladivostok population is approximately 600,000 .

\subsection{Study Design}

Totally 260 soil samples from sandpits and 155 samples from public parks were collected during July 2015 to December 2017; samples were collected monthly excluding January and February due to a snow cover preventing the sample collection. Each sample contained approximately 200 gr soil. Each sample was taken from different depths: from the top 5- $6 \mathrm{~cm}$ and from 10 to $15 \mathrm{~cm}$ below the surface.

\subsection{Parasitological Examinations of Soil}

Soil samples were analyzed using flotation sedimentation method. Soil samples $25 \mathrm{~g}$ each are mixed with water (1:2) in $50 \mathrm{~mL}$ centrifuge tubes, and centrifuged 1200 $\mathrm{rpm} \times 4 \mathrm{~min}$. Then the sediment is centrifuged again with $\mathrm{NaNO}_{3}(1 \mathrm{~L}$ water per $1 \mathrm{~kg} \mathrm{NaNO}$ ) added. Finally, the sediment was re-suspended in $50 \mathrm{~mL}$ saturated and poured into centrifuge tubes, which were filled to the brim, and the coverslip was superimposed. The samples were stored at the room temperature for $30 \mathrm{~min}$, the coverslip was removed onto a microscopic slide and examined for the presence of parasite eggs.

\subsection{Toxocara in Humans}

The incidence of Toxocara canis in humans was analyzed using Toxocara IgG antibody ELISA assay. Analyzes were provided in hospitals of Vladivostok, where the human patients admitted for the general health check routine. The prevalence was estimated using Software "analysis of population morbidity" (the Center of Hygiene and Epidemiology of Sverdlovsk Region, Russia). Incidence rates of toxocariasis were expressed as cases per 100000 person-years.

\subsection{Statistical Analyses}

Correlation between Toxocara spp. prevalence in soil and the incidence in humans were obtained via Pearson correlation test. The bivariate logistic regression was used for analyze the factors influenced on the Toxocara spp. presence in sandpits, where the Toxocara infection was dependent valuable and month and access for animals were factor valuables.

\section{Results}

\subsection{Toxocara spp. Prevalence in Sandpits}

Overall the annual prevalence of Toxocara spp. in sandpits varied between $20 \%$ - $23 \%$ in different years, the highest prevalence was registered in 2015 and 2016 year, and the lowest prevalence was recorded in 2017 year.

The highest prevalence was registered in summer season; the peak of the prevalence was recorded in June 2017 (0.7\%). The Toxocara spp. eggs were not found in December and rare found in March and April (Figure 1). The average number of Toxocara spp. varied from 0 to 220 eggs per $1 \mathrm{~kg}$ over the 3-years study period (Table 1 ). The prevalence of infective eggs in samples was higher in summer period, particularly in July (Figure 2). The bivariate logistic regression analyze was not showed the significant correlation between the presence of Toxocara spp. and month and presence of Toxocara spp. in sandpits and animals access for sandpits (Table 2).

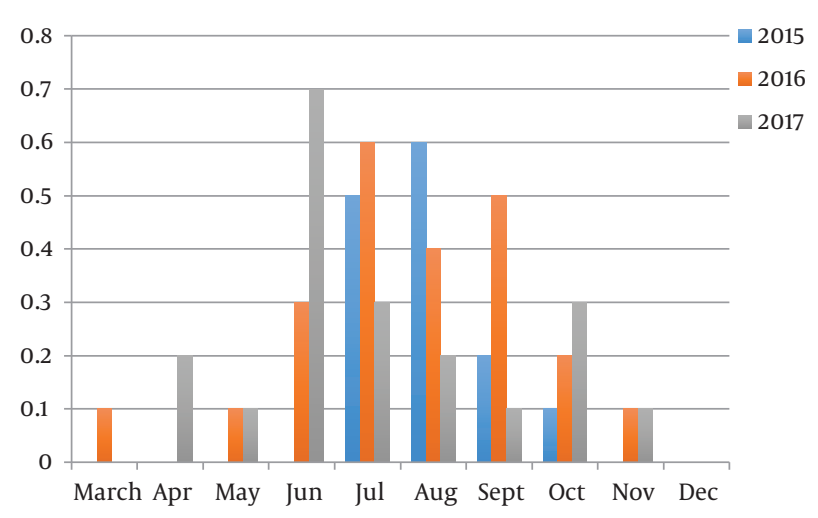

Figure 1. Monthly prevalence of Toxocara spp. eggs in sandpits during 3-year period

\subsection{Toxocara Prevalence in Public Parks}

Overall, the annual prevalence of Toxocara spp. in public parks varied between $25 \%$ - $42 \%$ in different years, the highest prevalence was registered in 2016 year, and the lowest prevalence was recorded in 2017 year. 

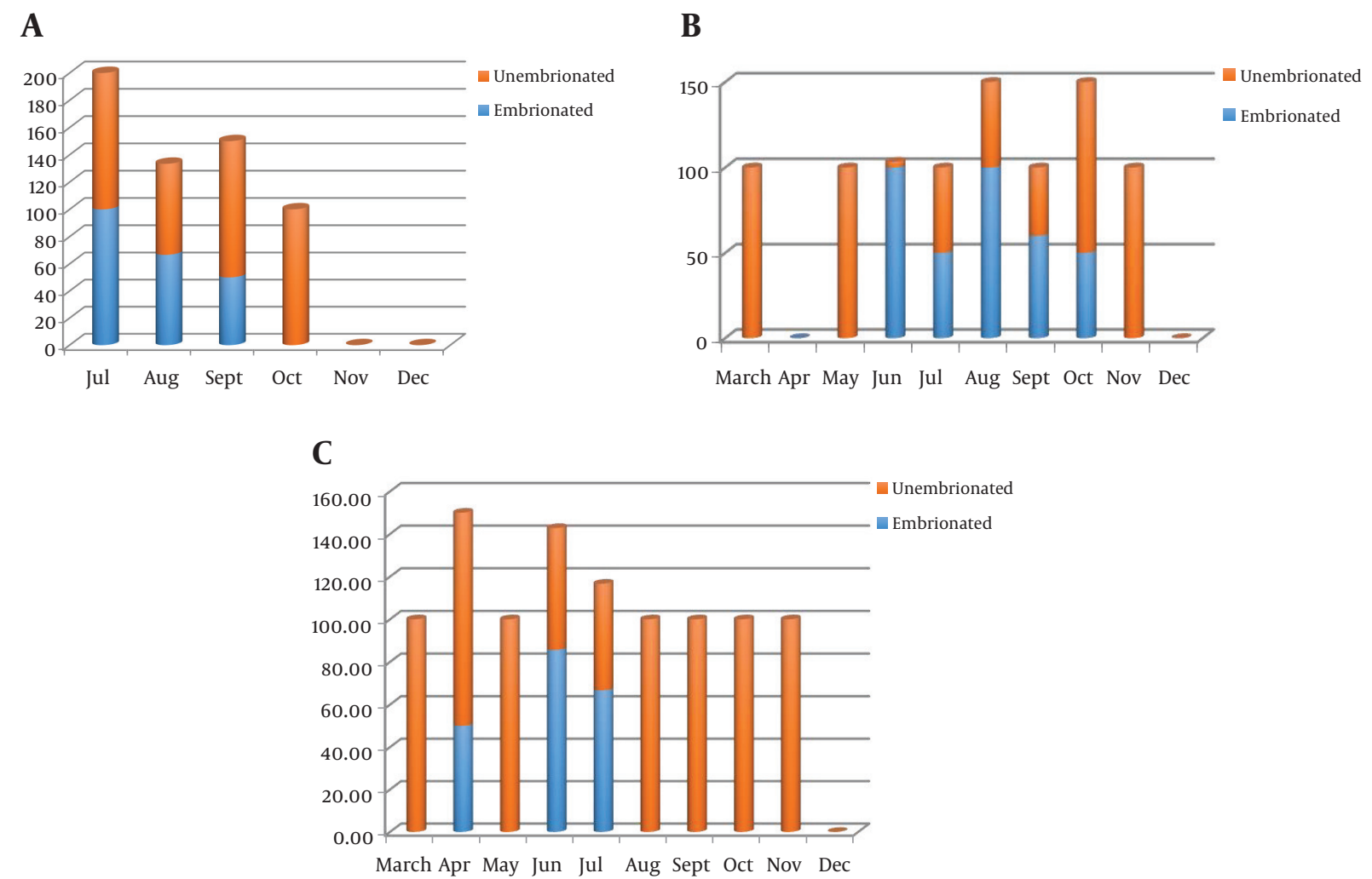

Figure 2. The prevalence of embryonated and unembryonated eggs in sandits. A, 2015; B, 2016; C, 2017.

\begin{tabular}{|c|c|c|c|}
\hline Season/Year & 2015 & 2016 & 2017 \\
\hline March & - & 0 & 20 \\
\hline April & - & 0 & 20 \\
\hline May & - & 20 & 20 \\
\hline June & - & 70 & 111.4 \\
\hline July & 216 & 70 & 70 \\
\hline August & 220 & 50 & 100 \\
\hline September & 140 & 44 & 160 \\
\hline October & 40 & 50 & 46.7 \\
\hline December & 0 & 0 & 0 \\
\hline Factor & Odds Ratio & $\mathbf{P}$ & [95\% Conf. Interval] \\
\hline Month & 0.97 & 0.54 & $0.88-1.07$ \\
\hline Access for animals & 1.71 & 0.49 & $0.37-7.88$ \\
\hline
\end{tabular}

The highest prevalence was registered in summer season; the peak of the prevalence was recorded in August
2016 (100\%). We have also never found Toxocara eggs in samples in December, however Toxocara spp. eggs were found in November and October.

\subsection{Toxocara Antibody in Human}

The annual incidence of toxocariasis in humans varied from 3.23 to $3.85 \%$; the highest incidence rate was registered in 2016, lowest in 2015. There is no common tend of season variation of the toxocariasis incidence in different years. As show in diagram the peak of infection was registered in June and November (0.63\%) in 2017; the highest incidence rate was recorded in February (0.67\%) in 2016; the high incidence rate was registered in February, August and November and it was $0.51 \%$ in 2015 (Figure 3). The positive monthly correlation between Toxocara incidence in soil and humans was registered in 2016 year (Table 3), whereas correlation was negative in other years.

\section{Discussion}

The results of present study indicated high environmental contamination of Toxocara spp. eggs, which lead to spreading of toxocariasis among humans (1). 


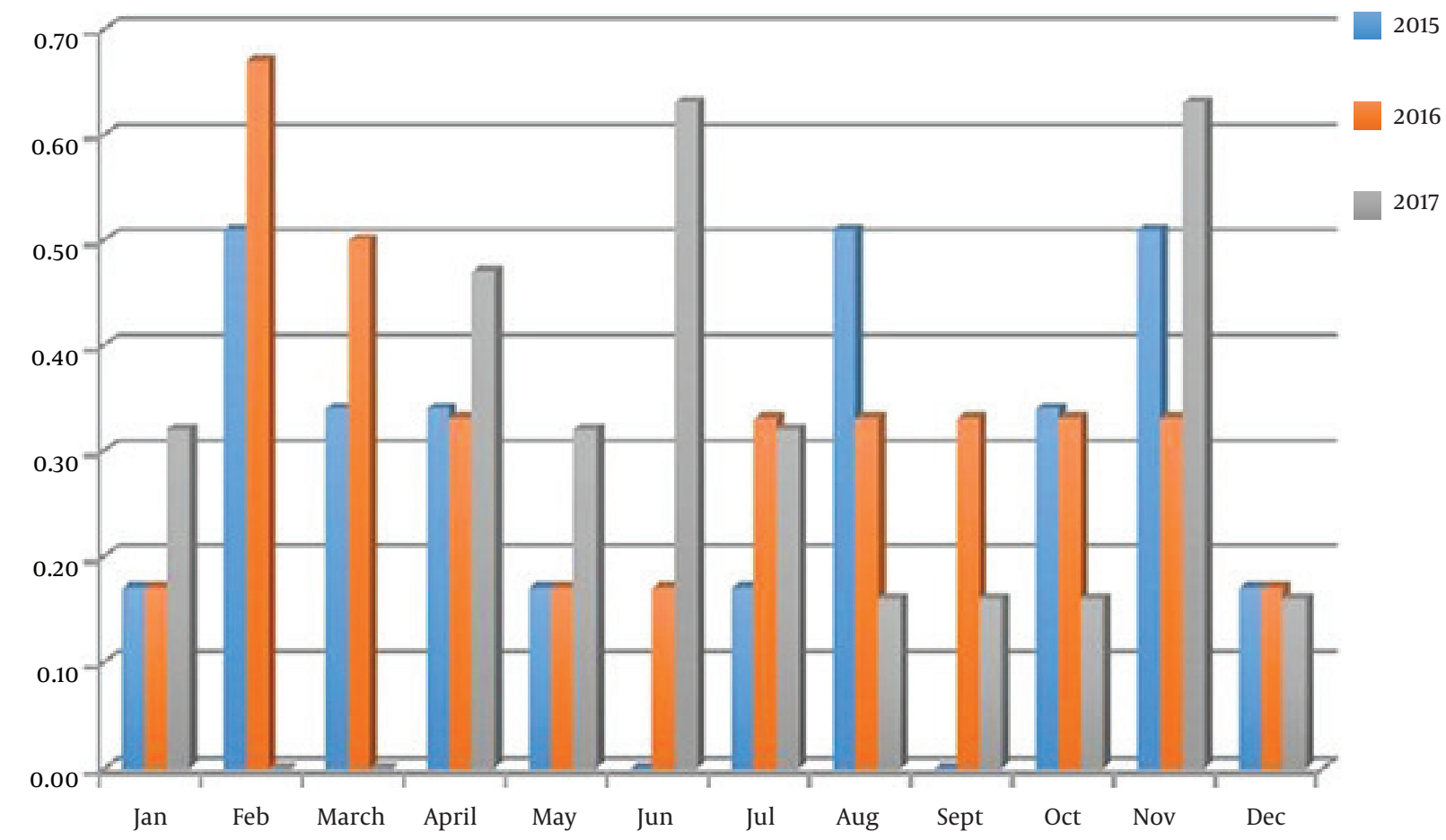

Figure 3. Toxocara incidence rates in humans

Table 3. The Correlation Between Monthly Toxocara Prevalence in Humans and Soil Samples

\begin{tabular}{ccc}
\hline Year & Human/Sandpits & Human/Parks \\
\hline 2015 & 0.3 & -0.2 \\
2016 & 0.1 & 0.01 \\
2017 & 0.6 & 0.1 \\
\hline
\end{tabular}

The overall prevalence of Toxocara spp. eggs in sandpits is comparable with the prevalence registered in Madrid, Spain (16.4\%), Lodz, Poland (15.7\%), and Hanover, Germany (23.2\%) (7-9).

Current study demonstrated, that the prevalence of Toxocara spp. embryonated eggs in sandboxes increased in summer season, and decreased in autumn. In Vladivostok totally three months such as July, August and September are the warmest months in the year (Table 4); the humidity is high and varies from $58 \%$ to $92 \%$ throughout the year. Relate to this both conditions including warm temperature and high humidity are necessary for third stage larva development and age survival. However Toxocara eggs are very resistant to harsh environment, rainfall and soil invertebrates such as slugs and earthworms can disperse eggs; eggs also are damaged at temperature as of $37^{\circ} \mathrm{C}(9,10)$. We can speculate that snowfall can also disperse Toxocara eggs, so we have not found Toxocara eggs in December. By contrast, Kleine et al. found the higher percentage of Toxocara spp. contamination in February, similar results were also demonstrated in other studies from Europe $(9,11,12)$. The cool and wet weather and high humidity in winter in Central Europe provides good condition for eggs survival and accumulation. In contrast, the significant season variation of Toxocara spp. contamination rates was not appeared in Northeastern Poland (12). The type of soil and meteorological factors can also influence on Toxocara spp. eggs survival and development (13).

We have no appeared the common tend for the season incidence of toxocariasis among humans. The stable incidence rates were appeared throughout the study period. The significant correlation appeared between Toxocara spp. contamination rates in sandboxes and parks and the Toxocara prevalence in humans suggests, that soil contaminated by Toxocara spp. eggs is a major source of spreading of human infection. However, our results are rube, because the tests were based on the detection of Toxocara canis IgG antibodies, which may persist many years after the infection. If proper hygiene measures are not implemented, environmental contamination can lead to infection (12). 


\begin{tabular}{|c|c|c|c|c|c|c|c|c|c|c|c|c|}
\hline Year/Month & Jan & Feb & March & April & May & Jun & Jul & Aug & Sept & Oct & Nov & Dec \\
\hline 2015 & -10.5 & -7.1 & 0.1 & 5.1 & 10.6 & 13.9 & 18.6 & 20.2 & 17 & 8.9 & -2.6 & -6.1 \\
\hline 2016 & -12.4 & -8.3 & 0.3 & 5.2 & 11 & 12.7 & 18.1 & 21.4 & 16.5 & 7.7 & -4.5 & -7.7 \\
\hline 2017 & -9.8 & -6.6 & 0.4 & 6.8 & 11.1 & 13.7 & 20.3 & 19.6 & 16.9 & 8.5 & -1.5 & -11.0 \\
\hline
\end{tabular}

\subsection{Conclusions}

The present study demonstrates season variation of Toxocara spp. eggs in soil samples. Warm temperature and high humidity in summer and autumn contributes to Toxocara eggs development. The limitation of our study is a method of Toxocara canis infection detection in human sera. The IgG titer may persist over the time, so the results of this parameter of rude. The PCR detection is method that is more sensitive, however it is rare provided in Russian hospitals.

Current study also demonstrates that it is necessary to promote preventive public health measures to avoid soil contamination.

\section{Acknowledgments}

The present study supported by the program of fundamental research of the Far Eastern Branch of Russian Academy of Sciences “Far East” 2018 - 2020, project 18-5-060.

\section{Footnotes}

Authors' Contribution: Tatyana Moskvina and Michail Shchelkanov obtain the data and write the MS. Tatyana Moskvina researched soil samples. Yulia Nesterova, Khomichuk Tatyana, and Galina Zakjarova presented the data about human toxocariasis. Irina Galkina and Marina Kiseleva edited the MS and work on diagrams and tables.

Clinical Trial Registration Code: There is no code (it is not a clinical study).

Conflict of Interests: Authors declare that they have no conflict of interests.

Ethical Approval: The study obtained ethics approval of the EC of Far Eastern Federal University (code: ID 20989).

Funding/Support: None.

Informed Consent: The study was used data of hospital laboratories, so all patients whose were informed.

\section{References}

1. Moreira GM, Telmo Pde L, Mendonca M, Moreira AN, McBride AJ, Scaini CJ, et al. Human toxocariasis: Current advances in diagnostics, treatment, and interventions. Trends Parasitol. 2014;30(9):456-64. doi: 10.1016/j.pt.2014.07.003. [PubMed: 25089038].
2. Mahmoudvand H, Taee N, Ebrahimzadeh F, Mirhosseini M, Faraji M. Seroprevalence and risk factors of toxocara canis infection in children (2-15 years old) referred to Health Centers of Lorestan Province, Iran. J Pediatr Infect Dis. 2017;13(1):20-4. doi: 10.1055/s-0037-1604325.

3. Zibaei M, Bahadory S, Cardillo N, Khatami AR. Soil contamination with eggs of toxocara species in public parks of Karaj, Iran. Int J Enteric Pathog. 2017;5(2):45-8. doi: 10.15171/ijep.2017.11.

4. Thomas D, Jeyathilakan N. Detection of Toxocara eggs in contaminated soil from various public places of Chennai CIty and detailed correlation with literature. J Parasit Dis. 2014;38(2):174-80. doi: 10.1007/s12639-012-0217-x. [PubMed: 24808647]. [PubMed Central: PMC4000375].

5. Mohd Zain SN, Rahman R, Lewis JW. Stray animal and human defecation as sources of soil-transmitted helminth eggs in playgrounds of Peninsular Malaysia. J Helminthol. 2015;89(6):740-7. doi: 10.1017/S0022149X14000716. [PubMed: 25273274].

6. Papajová I, Pipiková J, Papaj J, Čižmár A. Parasitic contamination of urban and rural environments in the Slovak Republic: Dog's excrements as a source. Helminthologia. 2014;51(4):273-80. doi: 10.2478/s11687-014-0241-8.

7. Otero D, Alho AM, Nijsse R, Roelfsema J, Overgaauw P, Madeira de Carvalho L. Environmental contamination with Toxocara spp. eggs in public parks and playground sandpits of Greater Lisbon, Portugal.JInfect Public Health.2018;11(1):94-8. doi:10.1016/j.jiph.2017.05.002. [PubMed: 28545900].

8. Blaszkowska J, Wojcik A, Kurnatowski P, Szwabe K. Geohelminth egg contamination of children's play areas in the city of Lodz (Poland). Vet Parasitol. 2013;192(1-3):228-33. doi: 10.1016/j.vetpar.2012.09.033. [PubMed: 23084538].

9. Kleine A, Springer A, Strube C. Seasonal variation in the prevalence of Toxocara eggs on children's playgrounds in the city of Hanover, Germany. Parasit Vectors. 2017;10(1):248. doi: 10.1186/s13071-017-2193-6. [PubMed: 28526064]. [PubMed Central: PMC5437484].

10. Macpherson $\mathrm{CN}$. The epidemiology and public health importance of toxocariasis: A zoonosis of global importance. Int J Parasitol. 2013;43(12-13):999-1008. doi: 10.1016/j.ijpara.2013.07.004. [PubMed: 23954435].

11. Mizgajska-Wiktor H, Jarosz W, Fogt-Wyrwas R, Drzewiecka A. Distribution and dynamics of soil contamination with Toxocara canis and Toxocara cati eggs in Poland and prevention measures proposed after 20 years of study. Vet Parasitol. 2017;234:1-9. doi: 10.1016/j.vetpar.2016.12.011. [PubMed: 28115175].

12. Kroten A, Toczylowski K, Kiziewicz B, Oldak E, Sulik A. Environmental contamination with Toxocara eggs and seroprevalence of toxocariasis in children of northeastern Poland. Parasitol Res. 2016;115(1):205-9. doi: 10.1007/s00436-015-4736-0. [PubMed: 26385468].

13. Gao X, Wang H, Li J, Qin H, Xiao J. Influence of land use and meteorological factors on the spatial distribution of Toxocara canis and Toxocara cati eggs in soil in urban areas. Vet Parasitol. 2017;233:80-5. doi: 10.1016/j.vetpar.2016.12.004. [PubMed: 28043392]. 